\title{
LAS IDEAS FILOSÓFICAS DE LOCKE SOBRE EDUCACIÓN
}

\section{Locke's Philosophical Ideas on Education}

\author{
Arturo Lucas Cabello \\ UNHEVAL-Huánuco.alucasc59@hotmail.com. \\ htpps://orcid.org/0000-0003-4429-8438
}

\begin{abstract}
RESUMEN
En este trabajo se analizan las ideas sobre educación que John Locke propuso hacia mediados del siglo XVII. Pues, según él la educación debe sustentarse en la virtud y en los valores morales, incluso por encima de la educación epistémica, pues los hombres son buenos o malos, útiles o no por la educación que reciben desde la familia o la escuela. Se extiende a lo largo de nuestro sistema educativo una preocupación por su calidad, problema que para Locke ya era central, pues decía si queremos el bien común, era necesario contar con docentes talentosos y sólidamente educados en cualidades morales. La pedagogía de Locke puede servirnos como marco propedéutico, para reflexionar sobre las grandes fracturas de nuestro sistema educativo, y proponer una educación sostenible e inclusiva.
\end{abstract}

\section{Palabras clave:}

John Locke, empirismo, educación, virtud, valores morales, ciencia, ejemplo.

\begin{abstract}
This paper tries to analyze the ideas about education that the philosopher of the tabula rasa, John Locke, proposed towards the middle of the 17th century. According to him, education must be based more on virtue and moral values than epistemic education, since men are good or bad, useful or not, because of the education they receive from the family or school. A concern for its quality spreads throughout our educational system, a problem that for Locke was already central, since he said that if we want the common good, it is necessary to have talented teachers who are solidly educated in moral qualities. Locke's pedagogy can serve as a propaedeutic framework to reflect on the great fractures of our educational system, and to propose a sustainable and inclusive education.
\end{abstract}

\section{Keywords:}

John Locke, empiricism, education, virtue, moral values, science, example. 


\section{Introducción}

John Locke, nació en Inglaterra en 1632, de padres calvinistas, estudió en el colegio de Westminster y la universidad de Oxford, fue miembro de la prestigiosa comunidad científica The Royal Society. Su vasta producción intelectual fue de mucha influencia, en el terreno de la filosofía de la educación, la filosofía moral, la filosofía política, la epistemología y la religión; por lo que Michael Ayers afirmaba que "Lo que Newton hizo por la física en el siglo XVII, Locke lo hizo por la filosofía. La revolución que forjaron estos dos gigantes estableció las bases intelectuales del mundo moderno" (Ayers, 1998, p.74).

Sus reflexiones sobre la educación del caballero quedaron consagradas en su texto Pensamiento sobre la educación (1694). También se le reconoce como "Locke teólogo", por su preocupación intelectual por la religión", la cual está fundamentada en un texto póstumo titulado La razonabilidad del cristianismo (1695), en su Ensayo sobre la tolerancia (1667) y su Carta sobre la tolerancia (1685). Locke por la influencia que ejerció su filosofía política en los movimientos civiles posteriores, es reconocido como el padre del liberalismo político, ideas que quedaron consagradas en sus Dos tratados sobre el gobierno civil (1690). Sin embargo, el mayor logro de Locke reside en el terreno de la epistemología, sus reflexiones sobre la ciencia están contenidas en el Ensayo sobre el entendimiento humano (1689), justamente a este tratado, debe nuestro autor, su mayor prestigio intelectual, en él profundiza y explica la concepción corpuscular de la Naturaleza y la ontología del conocimiento, realismo filosófico ${ }^{2}$, que no solo es resultado de la especulación sino del vínculo con las investigaciones que se hacían entonces en el campo de la física y la química emergente.

La influencia que recibió Locke, en su formación intelectual, fue, entre otras aristas, de la literatura filosófica y científica. Al cabo de algún tiempo se constituyó en el protagonista del desarrollo científico de la química, la física y la biología. Su relación con Newton fue fraternal, en la introducción a la Carta sobre la tolerancia se afirma que Locke y Newton eran muy amigos y que "Ambos eran profundamente admirados por el mundo intelectual...", (Locke, 2001, p. 10), añádase a este vínculo, la amistad académica más cercana que también tuvo con el padre de la química moderna, Robert Boyle.

\section{La educación en la filosofía de Locke}

El ambiente cultural del Reino Unido en el siglo XVII se sustentó en la sabiduría clásica, fue un siglo de ideas innovadoras; y uno de esos paradigmas que había entrado en crisis y fue reemplazado por otro, fue el de la educación, en este proceso innovador, nuestro filósofo, tuvo un protagonismo importante, cuyas ideas tuvieron enorme influencia en los postulados pedagógicos de Rousseau.

1 En los estudios introductorios a La razonabilidad del cristianismo, p. XXIII, se puede leer que "Locke fue un erudito y un hombre de letras, cuyo interés por la teología se expresa en la posesión de un volumen de libros al respecto casi cuatro veces superior al de filosofía"

2 Realismo. "El término entró en el uso filosófico con Kant para designar doctrinas de interés actual y no simplemente histórico. Fichte afirmó que 'la doctrina de la ciencia es realista porque 'demuestra que es absolutamente imposible explicar la conciencia de las naturalezas finitas si no se admite la existencia de una fuerza independiente de ellas, a ellas opuesta de la cual dependan en su existencia empírica' ". El realismo desde entonces ha sido calificado y definido de diversas formas, una de ellas es "el R. empírico, una versión un poco más complicada que reconoce por añadidura la posibilidad de una verificación y, por lo tanto, la confirmación y la refutación de nuestras propias hipótesis" en tanto "el R. científico, que admite la existencia de entidades no observables, como los electrones, los campos de fuerzas" sostiene Nicola Abbagnano en su Diccionario de filosofía. 
Para Locke, filósofo empirista, la mente era una tabula rasa, un papel blanco sobre el cual solo la experiencia puede escribir las ideas de sensación y a partir de ella establecer relaciones de ideas complejas y luego construir conocimientos abstractos. Precisamente el concepto de educación de Locke, con enorme influencia en la ilustración europea tiene el sello empirista, la educación se basa en la experiencia y a partir de ella se constituye en una herramienta que puede cambiar la mente y la acción del hombre en aras de una sociedad más abierta al pensamiento crítico $^{3}$. En la introducción a los Escritos pedagógicos de Hegel, se puede leer: "De acuerdo con ello, a la educación le estará reservado un papel primordial, que Locke mismo no duda en resaltar: Ique los hombres son el resultado de la educación] (Hegel, 1998, p.7). Es evidente que, para Locke, el progreso y la prosperidad de una nación depende de la buena educación de los hombres, éstos son buenos o malos, útiles o no por la educación que reciben:

"El educar bien a los niños, es de tal modo el deber y la misión de los padres, y el bienestar y la prosperidad de las naciones depende... de ello".

"...el modo de educar a la juventud, en relación con su diversa condición, es también el modo más fácil, breve y adecuado para producir hombres virtuosos, hábiles y útiles en sus distintas vocaciones..." (Locke 2012, p. 27).

Se sabe hoy que la educación desde sus orígenes es una función esencial de la familia y la comunidad. En el seno de la familia la educación del niño emerge de una acción elemental: el ejemplo ${ }^{4}$ que los padres muestran influye de manera directa en la formación del futuro ciudadano; Locke dice: "... para conseguir de sus hijos el respeto para él y para sus órdenes debe él mismo profesar una gran reverencia por su hijo. Maxima debetur pueris reverentia. No hagáis delante de él lo que queráis que haga por imitación" (Locke, 2012, p. 103). Según Locke incluso se debe tener sumo cuidado en elegir las amistades para los hijos, porque una mala influencia tiene la virtud de destruir todo lo bueno que la educación hizo por la buena crianza.

Este aporte de Locke es importante, porque nos permite volver a pensar en la familia, hoy absorbida por el consumismo tecnológico y sumida en la sobrevivencia espiritual y fáctica generando una ruptura dialógica entre los miembros de la familia cuya consecuencia en los hijos es un alma vacía, un presente sin futuro explícito. Otro elemento importante en la filosofía de la educación de Locke es el perfil del profesor. Según él, una buena educación no depende solo de un isomorfismo entre padre e hijo sino del

3 Jacques Boisvert, en su texto La Formación del Pensamiento Crítico, pág. 17, 18, 24 y 27, sostiene que este, se pueda abordado "desde tres ángulos complementarios: como una estrategia de pensamiento, como una investigación y como un proceso". "Para Kurfiss (1988), el pensamiento crítico es 'una investigación cuyo propósito es explorar una situación, fenómeno, pregunta o problema para elaborar una hipótesis o llegar a una conclusión". "Preguntarse sobre la necesidad de formar un pensamiento crítico parece un requisito para cualquier acción educativa". "Para Reboul (1984), toda verdadera enseñanza debe incluir la formación del pensamiento crítico, que aspire a favorecer el desarrollo de la autonomía de este pensamiento en los alumnos"

4 Luis Castro-Kikuchi, en su Diccionario ciencias de la educación, sobre el ejemplo explica: "En el ámbito educativo, tanto en la teoría como en la práctica pedagógicas, el ejemplo posee una gran tradición: al sustentar su importante valor, se insiste siempre en su enorme poder motivacional...el maestro no sólo es un orientador, es también, y en primera instancia...un modelo a imitar por el niño, un ejemplo a seguir... Wallon enfatiza en el hecho de que el maestro 'posee una autoridad social, intelectual y moral' " por lo mismo el maestro tiene "..la responsabilidad moral de crecer como persona para ser cada vez un mejor modelo" 
apoyo de un buen profesor. La educación moral y de las virtudes ${ }^{5}$ según Locke, no se restringiría a la moral familiar. En sociedades estructuralmente complejas, que precisamente se inició en la época de Locke, la educación recae en instituciones escolares como parte de un sistema más complejo, en tal sentido esta se extiende naturalmente al buen ejemplo del maestro, la conducta es un factor incisivo en la formación del niño, entonces no solo se educa en los libros; por el contrario, ella se fortalece sobre todo con el ejemplo, esto es con la autoridad moral del docente.

Locke dirá: ". . la conducta del padre ante el hijo debe extenderse a todos los que tienen autoridad sobre los niños, o a aquellos a quienes puedan profesar algún respeto" (Locke, 2012, p. 103). $\mathrm{Ni}$ la escuela, ni la familia son islas o archipiélagos en el acto formativo de la persona, estas son instituciones micro que pertenecen a otro sistema más grande: la sociedad. Entendida así la educación es una función sociocultural (Vygotsky) $\mathrm{y}$, por tanto, todas sus instituciones también educan. Entonces, estas no solo son infraestructuras, son mentes pensantes que actúan intersubjetivamente y de manera dinámica, si sus acciones estuviesen basadas en principios morales, estarían propiciando la construcción de una sociedad socialmente más armoniosa y empática, y no tan indiferente y corrupta como el que vive hoy el mundo.

El ejemplo es un método didáctico eficaz sobre todo cuando se trata de una educación en valores, el ejemplo de los padres, el maestro y los actores sociales, es de altísimo valor formativo, es el "currículum oculto" con resultados probados, que sin el mayor esfuerzo es "absorbido" por el inconsciente del niño, aquí cobra realidad la idea de que las palabras hacen ruido y el ejemplo convence, será por eso que Locke insiste en señalar que "...de todos los modos de instruir al niño, de formar sus costumbres, el más sencillo, el más fácil y eficaz es el de ponerle ante los ojos los ejemplos de las cosas que queréis hacerle practicar o evitar" (Locke, 2012, p. 116).

Retornemos a la dimensión del profesor, para comprender los bajos resultados educativos en el Perú, que son de preocupación, sobre todo si se observa el problema desde un contexto global. El bajo nivel de compresión lectora y resolución de problemas matemáticos, son indicadores que nos muestran el nivel educativo de los estudiantes peruanos, una realidad grave cuando se sabe que desde Tales de Mileto las matemáticas son fundamentales para la creación e innovación del conocimiento; el padre de la ciencia moderna, con razón habría dicho que, si él volviera a empezar, empezaría como Platón, por las matemáticas. Y de otro lado, el libro, habitante del tercer mundo popperiano, es determinante, porque de su entendimiento parte la mejor explicación racional del mundo y de ella emerge los problemas más importantes para innovar el conocimiento científico; sin embargo, esto está lejos de ser una realidad en el Perú, porque como dice Piscoya, tenemos profesores sin cultura pedagógica, que destacan sobre todo por su extremo pedagogicismo que radica en priorizar el cómo de la educación sobre el qué de la educación, es decir, convierte las disciplinas científicas en elementos subsidiarios. El precario aprendizaje del niño -continua Piscoya- se debe a que sus "profesores son formados durante

5 Sobre la virtud Ferrater Mora en su Diccionario de filosofía sostiene: "Siguió siendo común en la época moderna asociar 'virtud' con 'hábito'. Se trata de un hábito, o disposición, de obrar conforme a la intensión moral... La virtud es concebida, asimismo como el ánimo y el coraje de obrar bien". 
cinco años para que intenten 'enseñar' lo que, estrictamente, no saben" (Piscoya, 2005, p.95), cuando la realidad del mundo globalizado reclama docentes acreditados, por lo tanto, calificados y competentes. En un trabajo publicado por la PUCP Fernando D'Alessio sostiene que "...para alcanzar altos niveles de competitividad y soportar un crecimiento sostenible en el tiempo, es necesario disponer de capital humano calificado.", Es importante tener, precisa D'Alessio, "...docentes altamente capacitados, con desempeño docente acreditados y dotados con las competencias adecuadas para ... brindar una educación de calidad..." (D'Alessio, 2012, p.470) y no docentes con "deficiente formación profesional, que perpetúa la enseñanza basada en el copiado, el dictado y la repetición" (D'Alessio, 2012, p.407). El problema de la educación pasa también por el hecho de que los más dotados mentalmente aspiran a profesiones más liberales y de "prestigio" socialmente y no a la docencia. Este problema contemporáneo de la educación pública, cuasi carente de profesores talentosos, para Vincenzio padre de Galileo, ya era una preocupación, es por eso que, convenció a Galileo, para estudiar medicina a los 17 años en la universidad de Pisa, aunque luego renunció por las matemáticas apoyado por el matemático Ostilio Ricci. Poco después, este problema pasó a ser un tema central en la pedagogía de Locke, porque, igualmente, los hombres talentosos tenían poca inclinación por la docencia, sin embargo decía Locke que esto para el padre no debe ser óbice para procurar al máximo un profesor con cualidades para garantizar una buena educación para sus hijos, "...una persona prudente, moderada, discreta, en fin, que tenga por misión formarlas como conviene y preservado de todo mal. . . pienso -añade-, que este empleo exige mucha moderación, medida, ternura, celo y discreción" (Locke, 2012, p. 122).
Cualidades según Locke, difícil de encontrar todas juntas en un profesor; sin embargo, no hay que escatimar esfuerzos menos dinero para convencer a una persona capaz, si queremos educar al niño para su bienestar. La educación no solo es por el bienestar del individuo, también es por el bienestar común, que podría depender de un factor directo: la educación en la escuela; y de otro factor indirecto: la educación doméstica y de los actores sociales, que Locke llama con propiedad al primero educación doméstica y al segundo educación pública, si ambos evitan sus debilidades o "inconvenientes" podrán lograr una educación completa (Locke, 2012, p.103).

Locke tenía una admiración por la filosofía natural, igual por sus protagonistas. En el Ensayo sobre el entendimiento humano, John Locke, se refiere a Newton y a otros sabios de la época, como insignes arquitectos que van construyendo la ciencia, los mismos que serán admirados por la posteridad, en tanto el suyo es apenas una aspiración sincera por la verdad, por lo que "...no todos pueden esperar a ser un Boyle o un Sydenham" o luminarias "...como el gran Huygenius, el incomparable señor Newton" (Locke, 2013, p.10) que frente a ellos, añade Locke, su trabajo era solo la de un obrero de la ciencia, lo cual expresa la nobleza académica de Locke, aun siendo él un hombre cultivado en la ciencia, un médico exitoso, su inclinación por la química y la física una verdadera revelación del quehacer filosófico, aun así reiteraba una y otra vez su prioridad por la educación de la persona; es en este sentido, que para nuestro filósofo, la educación, no dependería solo del libro ni solo de la ciencia, así como el sastre - dirá Locke - que hace un traje a la moda o un maestro de baile que estiliza tus movimientos no te hace un hombre bien educado, igual, ni el libro ni la ciencia te 
educan; estas, socialmente se justifican si se construye sobre valores humanos; es en este sentido, se reafirma que se necesita profesores educados sólidamente en cualidades morales. Puesto que poco haría un talentoso matemático por una ciudadanía democrática que necesita más cultivar el espíritu en el "deber ser", y el pensamiento altruista.

Para formar...es necesario que el preceptor sea un hombre bien educado, ... Este es un arte que no se puede aprender ni enseñar en los libros. [La formación del joven no viene]. ...ni... siquiera agregándolo la ciencia, porque ésta, si no se proporciona bien, le hará más impertinente e intolerable en el trato. La educación es la que da brillo a las otras cualidades... Sin la educación, todas las demás cualidades no consiguen sino hacerle pasar por un hombre orgulloso, pedante, vano o tonto (Locke, 2012, p. 124).

\section{Educación y valores morales}

La educación en su condición formadora es quizá la única acción humana capaz de cambiar y hacer del hombre conciencia moral. Es capaz de forjar una mente innovadora, capaz de generar una nueva visión de la vida intersubjetiva y la Naturaleza, la educación en su condición formadora establece valores para crecer en comunidad tan heterogénea culturalmente como la nuestra, a su vez, puede generar competencias mentales como la ciencia que, si se inclinara por ese viejo ideario, el bienestar del hombre y el progreso humano estaría justificada por su humanismo. "La educación...no puede tener ningún otro fin que el de humanizarnos" dice Hegel (1998, p.9). El hombre es lo que es mediante la educación, es en este sentido que para Hegel "El hombre debe nacer dos veces, en cuanto natural y en cuanto espiritual" (1998, p.15). La educación también debemos entenderla como un proceso de aprendizaje y desaprendizaje de valores ${ }^{6}$, las sociedades evolucionan y con ella los valores. Si entendemos los valores morales como la forma correcta de actuar, entonces estos no son naturales sino adquiridos y las instituciones donde se aprende las primeras normas son en la familia y la escuela: "...el éxito del país se soportará en los niños y niñas que tengan acceso a una buena educación en el hogar y en la escuela con valores y principios éticos" (D'Alesso, 2012, p, 470). Sin embargo, casi podemos afirmar que la familia y la escuela se encuentran en crisis y no llegan a garantizar una formación moral, a decir de Piscoya: "La crisis de valores y de credibilidad que afecta a las instituciones [del país]" nos induce a una funesta entropía social.

En un mundo globalizado, con grandes rupturas sociales internas y externas, no solo es necesario el "el saber, es poder", es fundamental "el saber, empático"

6 Graciela Perrone y Flavia Propper en su Diccionario de educación, sostiene sobre valores, que es un "Conjunto de actitudes o atributos que describen el comportamiento de los individuos o las organizaciones. Los valores que se generan a nivel individual o colectivo conforman un sistema axiológico por el cual se rigen sus decisiones y sus actividades que se traduce en su ética. Los valores se forman con el ejemplo que se recibe desde el entorno familiar o institucional. La escuela es un espacio preciado para construir los valores ciudadanos que comienza a expresarse cuando el niño comienza a interactuar fuera de su entorno cultural primario". Augusto Salazar Bondy, en su texto Para una filosofía del valor suscribe: "Valorar, en un sentido propio, implica un compromiso, y una exigencia ante los demás. Exige coherencia, y apela a razones" ). "El operar sobre la realidad, el modificarla o alterarla, el construir algo en ella, así como el promover conscientemente conductas, el perseguir y alcanzar fines y el asumir determinadas consecuencias valiosas derivadas de nuestro comportamiento, son todos aspectos y formas de realización del valor" este es la realización del valor. (70) y cuando se refiere a Lo bueno moral, dice "Moralmente bueno es sólo un acto querido, una decisión tomada, una omisión voluntaria o una persona en tanto que quiere, hace u omite algo deliberadamente" (Salazar B.: p.24, 70, 155, 2010) 
Entonces resulta necesario fortalecer la dinámica entre el hemisferio derecho y el hemisferio izquierdo a través del desarrollo del "cuerpo calloso", porque el hombre no solo necesita del razonamiento matemático para vivir civilizadamente en democracia, sino también necesita de la empatía, por tanto es tan importante el "área de broca" como el "área de Werniker". El hemisferio derecho porque es el responsable de conectarnos con la dimensión inmaterial y espiritual, una de ellas los valores morales, logros que Locke no alcanzó a conocer, pero como ilustrado sabía que la educación en valores morales es igualmente importante, Locke dice "...pienso que puede afirmarse que todos los hombres con que tropezamos, ...son, buenos o malos, útiles o inútiles, por la educación que han recibido" (Locke, 2012, p. 31). Esta dimensión ética, ayuda a pensar si el hombre recibe una buena educación, basada en valores morales, podrá desarrollar su empatía, una visión del mundo integrado también con la necesidad del otro, distinto a uno, pero igual en dignidad, así se entiende si nuestra educación está basada en la moral, como dice Moore "... existe una fuerte convicción de que las enseñanzas éticas... son esenciales en la educación, y que esta no sería posible sin aquellas. De hecho, este punto de vista constituye una teoría acerca de la educación, es decir, la teoría de que la educación involucra necesariamente un contenido... moral" (Moore, 2007, p. 77).

Los valores morales son productos culturales, quizá además de la ciencia una de las más importantes para el hombre que aspira a vivir en una comunidad virtuosa y humanista. Sin embargo, ante nuestro abandono moral, los hombres han ido gestando, los códigos de ética, hechas para que el hombre tenga una conducta proba; sin embargo, este tendrá alcance limitado por más positivo que sea, porque la moral es el resultado de un proceso formativo y si carece de ello, el hombre ante la mínima oportunidad de lograr satisfacciones materiales por medios ilícitos lo hará. Rafael Termes dice: "Sin negar que tales códigos éticos puedan ser útiles, por muy acertados y detallados que sean, no lograrán el correcto funcionamiento de la empresa si en sus gentes está ausente el ejercicio prudencial de todas las virtudes morales" (Gonzales, 2014, p.57), esto implica que los códigos morales no son hechos biológicos determinados, es conciencia moral ${ }^{7}$.

El hombre, ya lo sostenía Aristóteles, es un ser gregario por naturaleza, su vida es una acción intersubjetiva permanente, nace y se desarrolla en torno a la cultura y la sociedad, nunca se excluye de ella, y mientras esté en ella sus acciones serán "buenas" o "malas", rechazadas o aceptadas, perjudicarán o ayudarán la convivencia mutua. Por ello añade Moore: "La moralidad... el comportamiento humano juzgado desde un punto de vista normativo; es decir, a lo que se debe hacer... la moralidad se aplica a las acciones que afectan los intereses y el bienestar no solo de nosotros sino también de los demás" (2007, p, 78).

La sabiduría socrática, también radica en haber considerado como vital la filosofía antropológica, hoy igualmente el "ethos reflexivo" reclama colocar al hombre nuevamente en el centro de nuestra reflexión, y hay la necesidad de reconstruir la ética desde la educación, y la necesidad

7 Rafael Termes, citado por Jesús Gonzáles, en su texto Corrupción, ética y moral en las administraciones públicas, p. 57, dice que frente la ausencia de la "...conciencia moral, fruto de la cultura del ser, frente a la cultura del disfrute, por muchas normas de comportamiento que existan y por muchas sanciones que se establezcan para los comportamiento, las normas saltarán hechas añicos cada vez que se presente la oportunidad de obtener, por medios torcidos la satisfacción de los apetitos". 
de recuperar la dignidad humana teniendo como eje la escuela, según Locke: "...las costumbres y las aptitudes de los hombres, son debidas a su educación" (Locke, 2012, p. 65). No cabe duda de que el bienestar del hombre comprende necesariamente el desarrollo económico, por tanto, implica esto cambiar nuestro pensamiento convergente por otro, el pensamiento divergente, para interpelar nuestra verdad y crear nuevos conocimientos abstractos, que con una tecnología pertinente puedan sumar nuestro bienestar fáctico, pero todo inspirado en consideraciones éticas. Precisamente la educación a los ojos de Locke tiene, ante todo, un carácter moral; tiene por objeto formar hombres que tengan conciencia de su dignidad, provistos de buenos hábitos, discretos más que instruidos. Locke pertenece a esta escuela de pedagogos que pone las cualidades morales por encima de los intelectuales (Locke, 2012, p. 66).

En su texto J. Locke: Ensayo sobre el entendimiento humano, Melendo sostiene: "Según Locke, la moral que propugna el Ensayo no solo evitará que los hombres se degraden al nivel de las bestias, destrozando por completo la sociedad en que viven; es también el remedio que liberará a la Humanidad del Príncipe de la Mentira y del rigor de la justicia divina" (Melendo, 1978, p. 257).

\section{Educación virtuosa}

En la filosofía de Locke, la educación moral, tiene como objeto el desarrollo de la virtud ${ }^{8}$. La virtud una de las dimensiones humanas, son aquellos valores adquiridos durante la vida, es un producto social, Aristóteles decía "...ninguna de las virtudes éticas se produce en nosotros por naturaleza" (Aristóteles, 2000, p. 160). Locke dirá que el hombre virtuoso es resultado de la escuela, en tanto Aristóteles decía mucho más antes que las virtudes que las adquirimos son el "resultado de actividades anteriores" de modo que "... practicando la justicia nos hacemos justos, practicando la moderación, moderados..." (Aristóteles, 2000, p.161).

El sistema pedagógico de Locke consideraba la educación virtuosa por encima incluso de la ciencia. Locke reconocía su admiración por la ciencia de su tiempo, aun los límites de la mente; admiraba la innovación revolucionaria del conocimiento científico, y sostenía que la pedagogía debe basarse en estos logros del conocimiento. Sin embargo, quizá influenciado por sus antecesores británicos, consideraba la importancia del conocimiento, en la medida que ésta esté del lado del progreso humano, el bienestar común. Es en este contexto, decía Locke, lo esencial y lo más difícil para la educación es la virtud. Todas las demás consideraciones y cualidades deben posponerse a esta. Este es el bien sólido y substancial que el preceptor debe convertir en objeto de sus lecturas y de sus conversaciones, y el arte de la educación debe llenar de ello el espíritu y, consagrarse a conseguirlo y no cesar hasta que los jóvenes sientan en ello un verdadero placer y coloque en ello su fuerza, su gloria y su alegría.

Para John Locke, la educación virtuosa era de primer orden, el hombre

8 "Vicente Hernández, en su texto La ética a Nicómaco de Aristóteles, página 82, sostiene que: Para Aristóteles existen dos clases o tipos de virtud, la "ética" y la "dianoética". Tras la primera se encuentra el concepto de éthos. Tras la segunda, una diánoia o reflexión intelectual". En el Diccionario de filosofía de Ezcurdia Híjar virtud significa "Disposición habitual para obrar moralmente bien". En Ferrater Mora, su Diccionario de filosofía, página, 3707, se lee que "La virtud es concebida...como el ánimo y coraje de obrar bien". "Kant trata la virtud como una 'fortaleza moral' a diferencia de la ausencia de la virtud, que es una 'debilidad moral'” y "destacó entre las virtudes, la fortaleza y la prudencia". 
virtuoso era el hombre que sentía un verdadero placer por la vida, "...cuanto más progreso haya hecho un niño en la virtud, más aptitud tendrá para adquirir las demás cualidades" como la gratitud, prudencia y responsabilidad (Locke, 2012, p. 102). El hombre virtuoso, es posible, por un lado, si se pone la educación en manos de hombres talentosos y de buena educación y, por otro, si es un logro básicamente de la casa; para dicho efecto los padres tienen que procurar un preceptor talentoso.

La educación de nuestra época ha cedido a favor de la educación racional, su prioridad es la de la razón, el estudiante de nuestro tiempo es instruido en el desarrollo de sus habilidades cognoscitivas de la ciencia y el pensamiento formal, es una educación cientificista con escaso resultado en nuestro contexto, pero aún es peor cuando se da en desmedro de la educación virtuosa diría Locke. Para nuestro filósofo, desarrollar un hombre virtuoso es la tarea más difícil que tiene que afrontar la educación, porque este no es una tarea ad hoc sino es una tarea esencial de la educación. Podría decirse que Locke, al sostener que la educación virtuosa, como la disposición habitual del hombre para obrar siempre moralmente bien, haya dejado entrever el desarrollo de la inteligencia emocional, ${ }^{9}$ como una herramienta que el joven puede manejar para lograr sus objetivos y metas no solo espirituales sino fácticos, Locke sostiene que: "La virtud es cosa más difícil de adquirir que el conocimiento del mundo, y, si la pierde el joven, es difícil recobrarla" (Locke, 2012, p. 98).
La lógica económica del mercado ha menguado nuestro interés por la dimensión ética y la educación virtuosa. Cuando todos creían que el laissez-faire era la luz del progreso social: "Los hechos se han encargado de desmentir la vieja idea smithiana de que una mano invisible conduce a los protagonistas de la vida económica a promover el bien común" (Gonzales, 2014, p.57). Nuestras políticas si de algo carecen es de virtudes, priorizan el "tener" antes que el "deber ser", es un mundo indiferente a la exclusión social: "En una sociedad que valora por encima de todo la productividad y la eficacia, la riqueza material y el individualismo posesivo - dice María Feria- no es de extrañar que los valores morales no encuentran sitio ni aplicación en las formas de vida que estamos construyendo" (Gonzales, 2014, p.43). La escuela debe educar hombres virtuosos, con "fortaleza moral" con vocación social que anhele la felicidad, pero sobre todo la felicidad social, es el anhelo de nuestro tiempo. Locke sostenía al respecto: "Lo que más reclama nuestro tiempo y nuestros ciudadanos asiduos es el establecer en su espíritu los principios y la práctica de la virtud y la buena educación" (2012, p, 99)

El lenguaje pedagógico del siglo XXI es la educación por competencias, fundamento epistémico que está en nubarrones para muchos docentes, pero está claro, es de enorme "debilidad moral", la educación científica se dice es el horizonte del bienestar común, seguro, pero si no está sobre pilotes éticos, solo fortalecerá el individualismo, compatible con el egoísmo. Locke, filósofo liberal ya decía que el gran cambio radica en la educación,

9 Daniel Goleman en su texto La inteligencia emocional pág. 13 se pregunta: "¿Qué factores entran en fuego, por ejemplo, cuando las personas que tienen un elevado coeficiente intelectual tienen dificultades y las que tienen un cociente intelectual modesto se desempeñan sorprendentemente bien? Yo afirmaría que las diferencia suele estar en las habilidades que aquí llamamos inteligencia emocional, que incluye el auto dominio, el celo y la persistencia, y la capacidad de motivarse uno mismo". Añade, "... que las posturas éticas fundamentales en la vida surgen de capacidades emocionales subyacentes." 
pero no solo en la educación científica, sino fundamentalmente en la educación moral; afirma que el "fundamento de la fortuna de su hijo [se halla] en la virtud y la buena crianza...único camino seguro y garantizado" (2012, p, 100). Nosotros compartimos el ideario de la educación como agente del gran cambio, pero una educación interculturalmente sostenible e inclusiva.

Por eso Locke en uno y otro lugar de su Pensamiento sobre la educación, subraya que una buena educación del niño debe "formar su espíritu en la virtud" (2012, p, 97), la inocencia y la modestia son las "virtudes que se aproximan más a las de un hombre útil y capaz, y lo prepara mejor" (2012, p. 98). El joven que haya sido educado en la virtud es un hombre hábil, precisamente "...los principios de justicia, de generosidad y de templanza, son los que hacen que un hombre sea hábil" (Locke, 2012, p.100). En cambio, la malicia, la violencia y la petulancia son vicios difíciles de desterrar en el alumno una vez adquiridas por ellos; así como el vicio es la sepultura del valor, para Locke la virtud será la llama que da vida a los valores morales, dice que: "el vicio es ya un mal más tenaz y más incurable y, por consiguiente, del que hay que defenderse en primer lugar", añade "el vicio es la tumba del valor" (2012, p. 98, 101).

La simpatía de Locke por la razón es más que evidente. El empirismo de Locke no es un puro fenomenalismo, es un empirismo racional, porque en sus sistemas, aun en oposición a Descartes, puso a la razón en un lugar relevante. El Ensayo reconoce a la mente como aquella que puede "ver" sus propias ideas y en La ley de la naturaleza explica "...que la razón es propiedad esencial del ser humano" (Locke, 2007, p. XI). Sin embargo, cuando justifica la virtud moral, inscribe su pensamiento dentro de la tradición cristiana más "ortodoxa", en esta línea, llega a considerar que si no fuese por la ley natural, el hombre, estaría avasallado por la codicia, el interés propio o la utilidad: "... sin la ley natural, no habría virtud ni vicio...Y como no habría nada que exigiese actuar de acuerdo con un deber, parece que el ser humano no estaría obligado a hacer nada más que lo que la utilidad o el placer lo aconsejan...Los términos 'recto' y 'virtuoso' desaparecerían por carecer de significado, o serían únicamente palabras vacías" (Locke, 2007, p.14). Solo por la ley natural sabemos quién es "virtuoso" y quién no lo es. Es en este sentido que ni las leyes positivas con su fuerza coercitiva ni los gobernantes con el poder que ostentan pueden ordenar cumplir una obligación moral, sino es en virtud de la ley natural.

Locke así se muestra partidario del cristianismo y consideró que esta era una "doctrina fundamentalmente ética" y gran parte de su preocupación fue encontrar demostraciones racionales a favor de estos principios éticos, aunque finalmente tuvo que aceptar que "... el evangelio era el gran documento en el que se contiene el más perfecto cuerpo de doctrina moral, dejando a un lado las consideraciones que tenía sobre la razón" (2007, p XIX).

Por encima de su simpatía por el cristianismo, la filosofía moral fue importante en el sistema de Locke, lo revela así sus textos: Ensayo sobre el entendimiento humano, La razonabilidad del cristianismo, Ley de la naturaleza, Ensayo sobre la tolerancia, igualmente su Pensamiento sobre la educación, trabajos importantes no solo en la historia del pensamiento sino relevantes para nuestra época, porque para revertir una sociedad inescrupulosa y que se maneja por la lógica de la felicidad hedonista, la 
filosofía moral de Locke podría ser una fuente válida para pensar razonablemente nuestra realidad. Tomás Melendo, autor del texto J. Locke: Ensayo sobre el entendimiento humano sostiene que las ideas de Locke pueden contemporizarse y Tzvetan Todorov en su texto Las morales de la historia, afirma que si el "bien común" es propósito de la filosofía de Locke se hace necesario retornar al pensamiento pedagógico del padre del liberalismo, aun con nuestros reparos, necesario si nos queremos librar de la anomia social que nos acosa, como describió Durkheim.

\section{Conclusiones}

La filosofía de la educación de Locke, con gran influjo empirista, interpreta críticamente la pedagogía normal de su época y propone frente a ella una pedagogía innovadora.

El tema central de la educación, según Locke, por encima de la filosofía natural, es la educación en valores, es esta la que garantiza la acción buena o mala del hombre en aras del bien común.

La pedagogía de Locke considera como definitorio, el rol de la familia y la escuela que, con el ejemplo, contribuye en la formación del niño como persona.

La pedagogía moral y virtuosa que Locke propuso podría ayudarnos a reflexionar y encontrar un derrotero a la crisis moral por la que atraviesa gran parte de nuestra sociedad, en aras de una educación sostenible e inclusiva.

\section{REFERENCIAS BIBLIOGRÁFICAS}

Abbagnano N. (2010) Diccionario de filosofía. México: Fondo de Cultura Económica.
Aguiló A. (2011) Libertad y tolerancia. Madrid: Ediciones Palabra.

Bertrand R. (1988) Diccionario del hombre contemporáneo. Uruguay: Imprenta Rosgal.

Buxarrais R. (2000) La formación del profesorado en educación en valores. Bilbao: Editorial Desclée.

Camps V. (1992) Historia de la ética. Barcelona: Editorial Crítica.

Castro-Kikuchi, L. (2005) Diccionario de ciencias de la educación. Lima: CEGURO EDITORES

D'Alessio, I. (2012) Planeamiento estratégico del sistema educativo del Perú. Lima: PERÚ OFFICE.

Del Águila y otros (2008) Democracia, tolerancia y educación cívica. Madrid: Ediciones UAM.

Ferrater M. (2004) Diccionario de filosofía. Barcelona: Editorial Ariel

Gonzales J. (2014) Corrupción, ética y moral en las administraciones públicas. Pamplona: Editorial Aranzadi.

Goleman D. (2000) La inteligencia emocional. Argentina: Ediciones B.

Hernández V. (1999) La ética a Nicómaco de Aristóteles. Madrid: Alianza Editorial.

Hegel, G.W.F. (1998) Escritos pedagógicos. México: FCE.

Locke J. (2001) Carta sobre la tolerancia. España: Ediciones Mestas. 
Locke, J. (2007) La ley de la naturaleza. Madrid: Editorial Tecnos

Locke, J. (2017) La razonabilidad del cristianismo. Madrid: Editorial Tecnos.

Locke J. (2012) Pensamiento sobre la educación. Madrid: Ediciones Akal.

Melendo, T. (1978) J. Locke: Ensayo sobre el entendimiento humano. Madrid: Editorial Magisterio Español.

Moore T.W. (2007) Filosofía de la educación. México: Trillas.
Neff, F. (1970) Filosofía y educación. Argentina: Editorial Troquel.

Perrone G. y Propper F. (2007) Diccionario de educación. Argentina: Alfagrama Ediciones

Piscoya, L. (2005) Cuánto saben nuestros maestros. Perú: Fondo Editorial de la UNMSM.

Salazar B. (2010) Para una filosofía del valor. España: FCE

Todorov, T. (1993) Las morales de la historia. Barcelona: Ediciones PAIDOS.

Fecha de recepción: 12-04-2020

Fecha de aceptación: 15-06-2020 